\title{
Implementation Of A Diffusion Convection Surface Evolution Model in WallDYN
}

\author{
K. Schmid ${ }^{*, a}$ \\ ${ }^{a}$ Max-Planck-Institut für Plasmaphysik, Boltzmannstraße 2, D-85748 Garching b. \\ München Germany
}

\begin{abstract}
In thermonuclear fusion experiments with multiple plasma facing materials the formation of mixed materials is inevitable. The formation of these mixed material layers is a dynamic process driven the tight interaction between transport in the plasma scrape off layer and erosion/(re-) deposition at the surface. To track this global material erosion/deposition balance and the resulting formation of mixed material layers the WallDYN code has been developed which couples surface processes and plasma transport. The current surface model in WallDYN can not fully handle the growth of layers nor does it include diffusion. However at elevated temperatures diffusion is a key process in the formation of mixed materials. To remedy this shortcoming a new surface model has been developed which, for the first time, describes both layer growth/recession and diffusion in a single continuous diffusion/convection equation. The paper will detail the derivation of the new surface model and compare it to TRIDYN calculations.
\end{abstract}

Key words:

PACS: 61.80.Jh, 52.25.Vy, 52.65, 52.40.Hf

PSI Keywords: Diffusion, DIVIMP, Erosion \& Deposition, Sputtering, Edge modeling

\section{Introduction}

One of the most important questions for future long pulse thermonuclear fusion experiments is the redistribution of the first wall material in time due

\footnotetext{
* K. Schmid

Email address: klaus.schmid@ipp.mpg.de (K. Schmid).
} 
to the combination of erosion, plasma-transport and (re-) deposition. The net balance between erosion and deposition determines the life time of the first wall and co-deposition with fuel species dominates fuel retention in machines, particularly in those with a low-Z first wall elements (e.g. Be in ITER) [1]. Additionally the surface composition that evolves in time is different from the initial material configuration since mixed material layers are formed by the continuous erosion/(re-) deposition. These mixed layers typically have quite different properties than the initial pure elements and can potentially hamper machine operation.

The global erosion/deposition balance and the formation of mixed layers are highly dynamic processes. They result form the tight coupling between transport of eroded impurities in the scrape off layer (SOL) plasma and the erosion/(re-)deposition processes at the first wall surface. However most current SOL codes operate under the assumption of a wall with static composition and most wall codes assume a static particle influx spectrum. Both of these assumptions do not hold in reality. Therefore the WallDYN code [2] has been developed which couples surface processes and plasma transport to track the global material erosion/deposition balance. In [2] is was shown that long range transport of material in a tokamak is a multi step process: Impurities are transported from one position to another by series of erosion/deposition/re-erosion and re-deposition steps. Therefore to describe the global erosion/deposition balance one must track these steps by following the evolution of the surface composition in time. In WallDYN this is done by describing the changes in the impurity influx at a given poloidal position due to erosion of impurities at all other poloidal positions as an algebraic equation system. The change in the surface composition resulting form this impurity influx is modeled by a simple ordinary differential equation (ODE) [3]. This approach allows one to couple plasma transport and surface dynamics in a single differential algebraic equation (DAE) system. This DAE system can be solved using linear multistep methods [4] which allows to truly (i.e. not iteratively, all processes occur simultaneously) couple different physical processes. As was also already pointed out in [2] this has numerous advantages over iteratively coupling SOL codes to Monte carlo (MC) surface codes or even to Molecular Dynamics (MD) and Density Functional Theory codes: Iterative coupling always occurs on different time scales and the error propagation during iterative coupling can be considerable. Also the continuous description does not suffer from sampling 
artifacts like MC codes (e.g. TRIM) do for incident particle spectra with very small flux fractions.

It should however also be noted that the continuous descriptions of physical processes have the disadvantage that the required rate determining parameters like sputter or reflection yields, with their potentially complex composition dependence, must be included in the models in a parameterized way. As was shown in [2] this is possible and allows to include the experimental data from $\ddot{2} 0$ yearsöf plasma wall interaction research and the output of sophisticated codes in WallDYN. Therefore WallDYN is not meant to replace MD, MC or DFT methods but is a tool to properly include their output in global material transport simulations.

The current surface model in use in WallDYN only tracks the surface composition evolution but it is well known that mixed material formation, in particular at elevated temperatures, is strongly influenced by diffusion. Further the current surface model does not fully handle layer growth/recession which is important to properly model co-deposition with fuel species. Following the WallDYN concept a continuous description of layer growth/recession and diffusion is required to be able to describe all processes in a DAE system. The current approaches to model thickness changes in a surface due to erosion/deposition typically involve discrete histograms of layers whose thickness and number are changed to model layer growth and recession. However this discrete description can not in a clean way (i.e. non iteratively) be coupled to diffusion which by nature is a continuous process described by Fick's second law. Therefore a new surface model was developed which for the first time describes both layer growth and recession together with diffusion in a single, continuous partial differential equation.

The paper will describe in detail the derivation of the PDE for the new surface model and how it is integrated into the WallDYN DAE system approach. Then the model will be compared to TRIDYN [5] which is the current standard to describe surface composition changes due to sputtering.

\section{Model Description}

The change in thickness of a sample during erosion and implantation of elemental species is due to a relaxation of the total local number density $\rho^{T O T}$. 
When material is net deposited (implanted) $\rho^{T O T}$ increases by $\Delta \rho^{T O T}$ whereas when material is net eroded $\rho^{T O T}$ decreases by $\Delta \rho^{T O T}$. As a response to this change in $\rho^{T O T}$ the system relaxes either by reducing the thickness by $\delta x$ (for $\Delta \rho^{T O T}<0$ ) or increasing the thickness by $\delta x$ (for $\Delta \rho^{T O T}>0$ ). A local change in thickness by $\delta x$ at position $\mathrm{x}$ within the surface can be seen as a movement of all positions $\tilde{x}>x$ in the surface if one assumes the surface at $\mathrm{x}=0$ to be the origin of the fixed frame of reference. This movement can be described by a convective term in the one dimensional material conservation equation 1

$$
\begin{aligned}
\frac{\partial \rho_{i}}{\partial t} & =-\frac{\partial}{\partial x}\left(v \rho_{i}\right)+\Phi_{i}+\frac{\partial}{\partial x}\left(D\left(\rho_{i}\right) \frac{\partial \rho_{i}}{\partial x}\right) \\
\rho_{i}(x, t) & =\text { Number density of species i at position x and time } \mathrm{t}\left(\frac{\#}{m^{3}}\right) \\
v(x, t) & =\text { Convection velocity field }\left(\frac{m}{s}\right) \\
\Phi_{i}(x, t) & =\text { Change in number density due to erosion/deposition }\left(\frac{\#}{m^{3} s}\right) \\
D\left(\rho_{j}\right) & =\text { Composition dependent diffusion coefficient }\left(\frac{m^{2}}{s}\right)
\end{aligned}
$$

To derive an expression for $v(x, t)$ one has to make an assumption about how the material relaxes after a change in $\rho^{T O T}$. To describe this relaxation one must make an assumption about the equilibrium number density of the system for a given composition, described by the atomic concentrations. For WallDYN the same approach is used as in TRIDYN [5] to assign a number density to a certain mixture of species. TRIDYN assumes that the volume $V_{i}$ per atom $\left(\Leftrightarrow \frac{1}{\rho_{i}}\right)$ of species $\mathrm{i}$ is the same as in the pure element. While this appears to be a rather coarse approximation the typical deviation for the actual $V_{i}$ found in a given compound is in the order of $10-20 \%$. This assumption of constant per atom volume can be used to calculate the relaxed density of a mixture $\rho^{M I X}$ as in eq. 2. 


$$
\begin{aligned}
\frac{1}{\rho^{M I X}} & =\sum_{i=1}^{N_{\text {Elem }}} \frac{c_{i}}{\rho_{i}^{\text {PURE }}} \\
c_{i} & =\frac{\rho_{i}}{\sum_{j=1}^{N_{\text {Elem }} \rho_{j}}} \\
c_{i} & =\text { Atomic concentration of species } \mathrm{i} \\
N_{\text {Elem }} & =\text { Number elemental species in the system } \\
\rho_{i}^{\text {PURE }} & =\text { Density of pure species } \mathrm{i}
\end{aligned}
$$

To derive an expression for $v(x, t)$ based on eq. 2 we first consider a single layer of initial thickness $\Delta x$. This layer contains $N_{E l e m}$ species with densities $\rho_{i}$ and corresponding concentrations $c_{i}$. This layer is now subject to a homogeneous change (over $\Delta x$ ) in the number densities $\Delta \rho_{i}$ corresponding to change $\Delta N_{i}=$ $\Delta \rho_{i} A \Delta x$ in the number of atoms in the layer. Where A $\left(m^{2}\right)$ is the surface area of our layer over which all processes are assumed to occur homogeneously. The layer now changes its thickness by $\delta x$ to relax its density to the $\rho^{M I X}$ corresponding to its current concentrations $c_{i}$ of the elements in the layer as shown in eq. 4.

$$
\begin{aligned}
\frac{A(\Delta x+\delta x)}{\sum_{i=1}^{N_{E l e m}} N_{i}+\Delta N_{i}}=\frac{1}{\rho^{T O T}} \equiv & \frac{1}{\rho^{M I X}} \\
= & \sum_{j=1}^{N_{E l e m} \frac{N_{j}+\Delta N_{j}}{\sum_{i=1}^{N_{E l e m} N_{i}+\Delta N_{i}}}} \\
\rho_{j}^{P U R E} & \text { Number of atoms of species k in layer of width } \Delta x \\
& \text { and area A }
\end{aligned}
$$

By utilizing $\Delta N_{i}=\Delta \rho_{i} A \Delta x$ and $N_{i}=\rho_{i} A \Delta x$ eq. 4 simplifies to eq. 5 .

$$
\Delta x \sum_{i=1}^{N_{\text {Elem }}} \frac{\Delta \rho_{i}+\rho_{i}}{\rho_{i}^{P U R E}}=\Delta x+\delta x
$$

Based on eq. 5 we now make the transition from a single layer over which the changes occur homogeneously over depth to a target of thickness $x^{*}$ where the changes in density are spatially distributed over depth (i.e. $\Delta \rho_{i} \equiv \Delta \rho_{i}(x, t)=$ $\left.\Phi_{i}(x, t) \delta t\right)$. This amounts to changing the multiplications by $\Delta x$ to integral 
expressions as shown in eq. 6 .

$$
\int_{0}^{x^{*}}\left(\sum_{i=1}^{N_{E l e m}} \frac{\Phi_{i}(\tilde{x}, t) \delta t+\rho_{i}(\tilde{x}, t)}{\rho_{i}^{P U R E}}\right) d \tilde{x}=x^{*}+\delta x
$$

After some transformations and using the fact that $\sum_{i} \frac{\rho_{i}}{\rho_{i}^{P U R E}}=1$, which follows from eq. 2 , one obtains eq. 7 from eq. 6

$$
\int_{0}^{x^{*}}\left(\sum_{i=1}^{N_{E l e m}} \frac{\Phi_{i}(\tilde{x}, t)}{\rho_{i}^{P U R E}}\right) d \tilde{x}=\frac{\delta x}{\delta t} \equiv v\left(x^{*}, t\right)
$$

Eq. 7 describes the motion of an arbitrary position $x^{*}$ within a target due to changes in density at positions $x<x^{*}$ and is the required convection velocity in eq. 1.

Eq. 6 only includes density changes due to erosion \& deposition via $\Phi_{i}(x, t)$. However in principle also diffusion can lead to a density depending on the choice for $D\left(\rho_{j}\right)$. In its current version the model only allows diffusive processes which do not significantly change the local density. From mass balance considerations (i.e Fick's second law) this includes all interdiffusion processes where all species have the same, composition dependent, diffusion coefficient. While this may appear a coarse limitation at first, such interdiffusion processes can be used to model e.g. interdiffusion of $\mathrm{C}$ and $\mathrm{W}[6]$ or Be and $\mathrm{W}$ [7].

Inserting eq. 7 into eq. 1 one obtains the final governing equation for the new diffusion convection surface model in WallDYN, eq. 8

$$
\frac{\partial \rho_{i}}{\partial t}=-\left(\rho_{i} \sum_{j=1}^{N_{E l e m}} \frac{\Phi_{j}}{\rho_{j}^{P U R E}}+\frac{\partial}{\partial x} \rho_{i} \sum_{j=1}^{N_{E l e m}} \frac{\int_{0}^{x} \Phi_{j} d \tilde{x}}{\rho_{j}^{P U R E}}\right)+\Phi_{i}+\frac{\partial}{\partial x}\left(D\left(\rho_{i}\right) \frac{\partial \rho_{i}}{\partial x} 8\right)
$$

Equation 8 is an integro partial differential equation. To use it in the WallDYN DAE system it must be converted to a system of coupled ODEs. This is achieved by applying the method of lines (MOL). In the MOL approach the right hand side (RHS) of eq. 8 is converted to an algebraic expression whereas the time derivative on the left hand side (LHS) remains. To convert the RHS to an algebraic expression first a grid is chosen that discretises the depth 
coordinate. Based in this grid the derivatives are approximated by upwind finite differences [8] and the integrals using the trapezoidal rule. After inserting the resulting coupled ODEs in the WallDYN DAE, the complete system can be solved using an implicit backward differentiation (BDE) solver [4] which is capable of handling DAEs.

\section{TRIDYN comparison}

Equation 8 is not specific to erosion deposition modeling but is generally applicable to $1 \mathrm{D}$ systems subject to thickness changes due to the density relaxations. To make it applicable to model erosion/deposition due to plasma impact an expression for $\Phi_{i}(x, t)$, the change in number density of species i at position $\mathrm{x}$, has to be specified. For the first tests of the model a comparison with the TRIDYN Monte Carlo code was chosen. To that end eq. 9 was used for $\Phi_{i}(x, t)$.

$$
\begin{aligned}
\Phi_{i}(x, t) & =\Gamma_{i}^{I n}\left(1-R_{i}\left(E_{i}\right)\right) \xi_{i}^{D e p}(x)-\left(\sum_{j=1}^{N_{\text {Elem }}} Y_{i, j}\left(E_{j}\right) \Gamma_{j}^{I n}\right) c_{i}(x, t) \xi_{i}^{\text {Ero }}(g) \\
\xi_{i}^{\text {Dep }}, \xi_{i}^{\text {Ero }} & =\text { Spatial distribution of erosion, deposition } \\
\Gamma_{i}^{I n} & =\text { Incident flux of species } \mathrm{i} \\
Y_{i, j}\left(E_{j}\right) & =\text { Sputter yield of element i by species } \mathrm{j} \text { at incident energy } E_{j} \\
R_{i}\left(E_{i}\right) & =\text { Reflection yield of element i by at incident energy } E_{j}
\end{aligned}
$$

The goal of the comparison with TRIDYN was to test whether the convection velocity term in eq. 8 would properly describe the growth or recession of layers. From now on results determined by solving eq. 8 will be referred to as WallDYN 2.0 results. To avoid ambiguities due to sputter and reflection yields the bombardment of Be by an incident flux of Be $+\mathrm{D}$ (for a net deposition case comparison) and by an incident flux of Be + Ar (for a net erosion case comparison) was simulated both by WallDYN 2.0 and by TRIDYN. The recycling species D and Ar only entered as eroding species an were not accumulatd in the target. They were included in the tests to assure that it would also work with multiple incident species which is important for incorporating the new surface model in WallDYN. Also switching from D to Ar allowed to switch 
from net deposition to a net erosion conditions without any further changes to the system. The sputter and reflection yields that enter as input in eq. 9 were taken from the respective TRIDYN runs. The parameters for the TRIDYN runs are summarized in table 1.

In Fig. 1 the result of the bombardment of Be with $\mathrm{Be}+\mathrm{D}$ as calculated by TRIDYN and by WallDYN 2.0 are compared. The match between the Monte Carlo code TRIDYN and the continuous description in WallDYN 2.0 is excellent.

In Fig. 2 a comparison of a TRIDYN and a WallDYN 2.0 calculation of the bombardment of a Be layer on Be by Be + Ar is shown. Exchanging D by Ar compared to Fig. 1 results in net erosion of the initially $5 \mathrm{~nm}$ thick Be layer. The match between WallDYN 2.0 and TRIDYN is very good for low fluences but at high fluences significant deviations occur. In particular the WallDYN 2.0 solution results in rather peaked near surface depth profile whereas the TRIDYN solution yields a rather flat profile. The reason for this lies in the lack of ion-beam mixing in the WallDYN 2.0 calculation which would normally lead to an intermixing of the near surface material thus "smoothing" the concentration profiles. This discrepancy is not a principal problem of the diffusion convection approach but is due to the imperfect description of the erosion process in eq. 9.

In Fig. 3 the convection velocity field for the net erosion and deposition case are shown. Negative velocities mean that material is moved towards the surface to compensate erosion losses whereas positive velocities mean that material is moved to the right out of the observed target volume.

The good match of WallDYN 2.0 with TRIDYN supports the choice of a Diffusion Convection approach for the new surface model for WallDYN. It is now for the first time possible to truly (non iteratively) model the simultaneous sputter erosion/deposition and diffusion in a multi species target.

\section{Conclusions}

The WallDYN approach allows to model complex coupling between plasma transport and surface PWI processes leading to a global erosion/deposition balance and the formation of mixed materials. The fundamental WallDYN paradigm is to truly couple the processes on the same time scale (i.e not it- 
eratively). This makes it necessary to describe all processes in a continuous form (PDEs, ODEs and algebraic equation systems) such that they can be combined in a large DAE system which can then be solved using linear multi step methods. This allows to include the output of sophisticated surface codes (e.g. MD or DFT) but also experimental data in a global transport code.

In order to be able to properly handle layer growth and recession and to include diffusion during mixed material formation a new WallDYN surface model has been developed. It is based on the diffusion convection material balance equation and allows for the first time to model the simultaneous sputter erosion/deposition and diffusion in a multi species target. First tests comparing it to TRIDYN calculations show that modeling layer growth and recession by a convective terms works very well. Further development is needed for the proper description of erosion to also include the effects of ion beam mixing. 
Figure \& Table captions

Fig. 1

Comparison of the WallDYN 2.0 surface model with a TRIDYN calculation of the bombardment of a Be surface by an incident flux of Be $+\mathrm{D}$. Due to net deposition a Be layer is growing.

Fig. 2

Comparison of the WallDYN 2.0 surface model with a TRIDYN calculation of the bombardment of a Be surface by an incident flux of $\mathrm{Be}+\mathrm{Ar}$. Due to net erosion the initial Be layer is receding.

Fig. 3

The convection velocitiy field in eq. 8 for net deposition and net erosion conditions.

Table 1

Input parameters in the TRIDYN runs. $\Phi$ denotes the fluence and $\varepsilon_{X}, E_{X}$ the fraction/energy of species $\mathrm{X}$ in the incident flux. 


\section{Tables}

\begin{tabular}{|c||c|c|c|c|c|}
\hline Case & $\Phi\left(m^{-2}\right)$ & $\varepsilon_{B e}$ & $\varepsilon_{A r / D}$ & $E_{B e}(\mathrm{eV})$ & $E_{A r / D}(\mathrm{eV})$ \\
\hline $\mathrm{Be}+\mathrm{Ar}$ & $5^{17}$ & 0.1 & 0.9 & 500 & 300 \\
$\mathrm{Be}+\mathrm{D}$ & $10^{18}$ & 0.5 & 0.5 & 500 & 500 \\
\hline
\end{tabular}

Table 1 
Figures

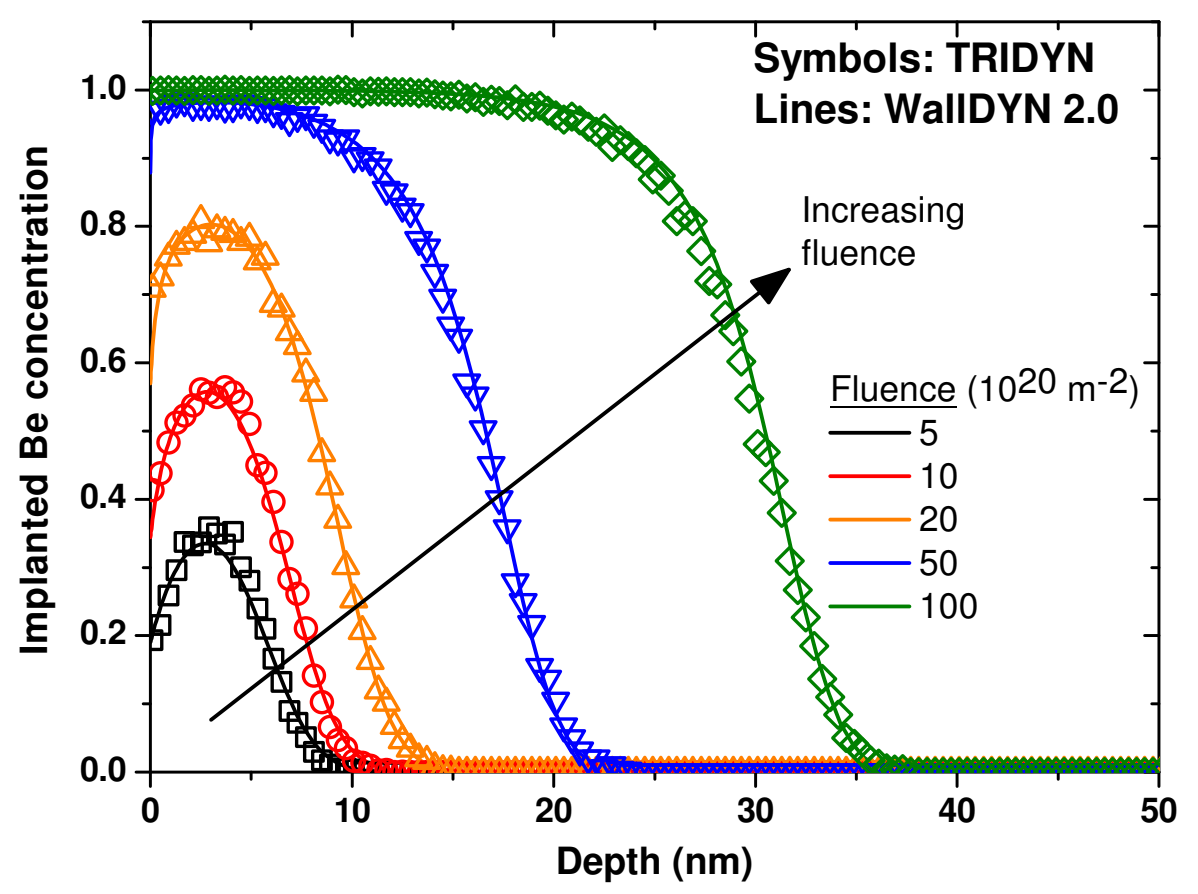

Fig. 1. 


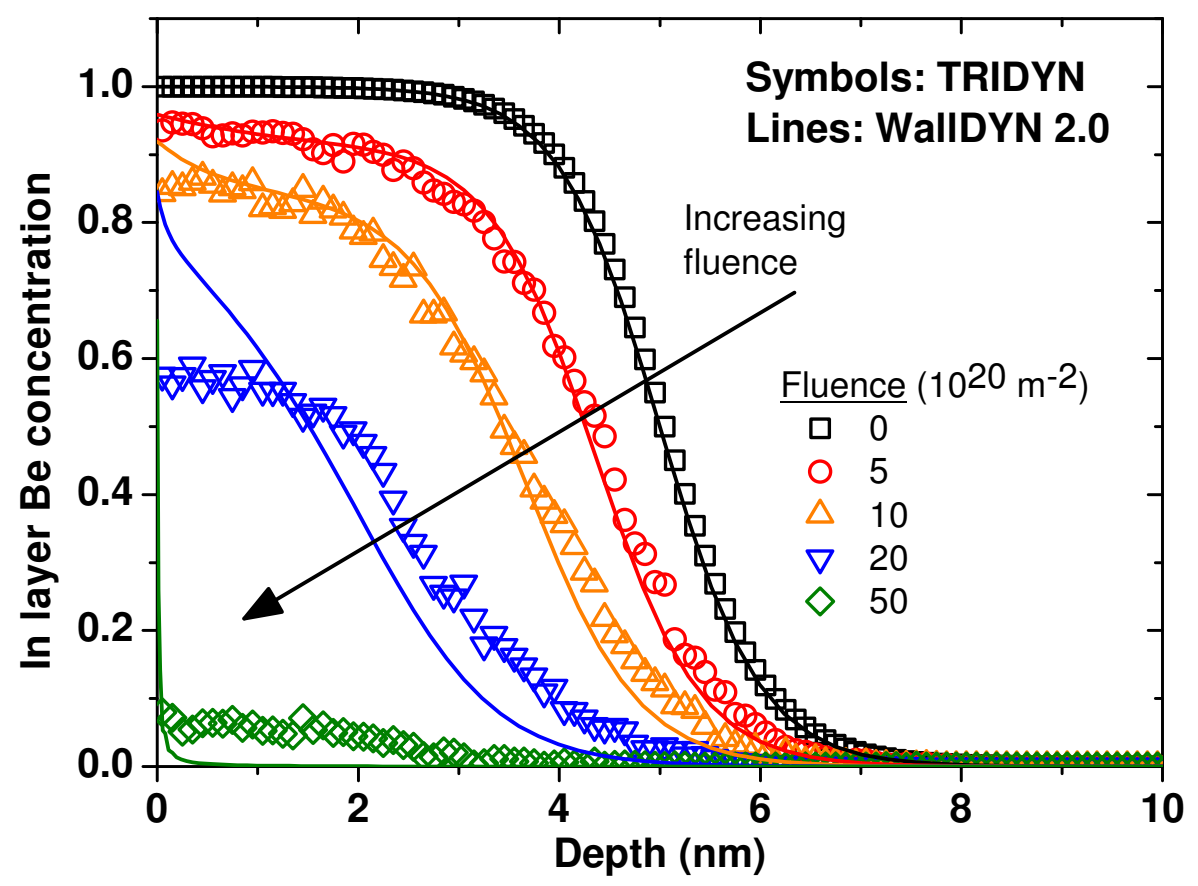

Fig. 2. 


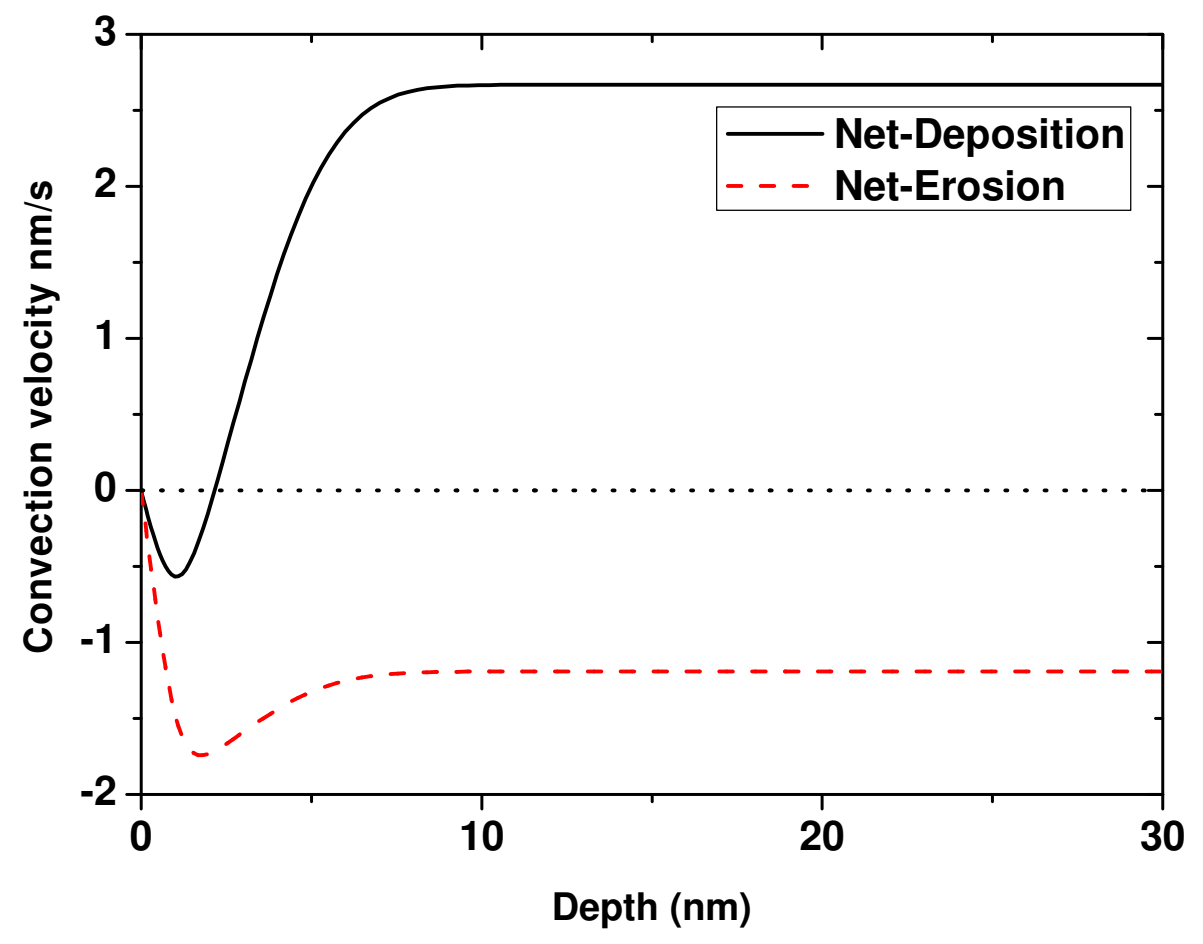

Fig. 3. 


\section{References}

[1] J. Roth, E. Tsitrone, A. Loarte, T. Loarer, et. Al., J. Nucl. Mater. 390-391 (2009) 1.

[2] K. Schmid, M. Reinelt, K. Krieger, J. Nucl. Mater. 415 (2011) 284.

[3] K. Schmid, M. J. Baldwin, R. P. Doerner, D. Nishijima, Nuclear Technology 159 (2007) 238.

[4] A. C. Hindmarsh, P. N. Brown, K. E. Grant, S. L. Lee, R. Serban, D. E. Shumaker, , C. S. Woodward, Trans. Math. Softw. 31 (2005) 363.

[5] W. Möller, W. E. und J. P. Biersack, Computer Physics Communications 51 No. 8 (1988) 355.

[6] K. Schmid, J. Roth, J. Nucl. Mater. 302 (2002) 96.

[7] K. Schmid, A. Wiltner, C. Linsmeier, Nucl. Inst. Meth. B. B219-220 (2004) 947.

[8] R. Courant, E. Isaacson, M. Rees, Comm. Pure Appl. Math. 5 (1952) 243. 